\title{
Nanodome coins for intracellular surface-enhanced Raman
}

\section{spectroscopy}

\author{
Pieter Wuytens ${ }^{\mathrm{a}, \mathrm{b}, \mathrm{c}}$, Winnok De Vos ${ }^{\mathrm{b}, \mathrm{d}}$, Andre Skirtach ${ }^{\mathrm{b}, \mathrm{c}}$, Roel Baets ${ }^{\mathrm{a}, \mathrm{c}}$ \\ ${ }^{a}$ Photonics Research Group, Ghent University-imec, Ghent, Belgium \\ ${ }^{\mathrm{b}}$ Department of Molecular Biotechnology, Ghent University, Ghent, Belgium \\ ${ }^{\mathrm{c} C}$ Center for Nano- and Biophotonics, Ghent University, Ghent, Belgium \\ ${ }^{\mathrm{d}}$ Dept. Veterinary Sciences, University of Antwerp, Antwerp, Belgium \\ pieter.wuytens@ugent.be
}

\begin{abstract}
Surface-enhanced Raman spectroscopy offers a tremendous potential for intracellular sensing, because it combines fingerprint specificity with sensitive signals. However, the colloidal gold nanoparticles used for this purpose lead to strong spatiotemporal variations in intracellular SERS experiments. This unpredictable behaviour, which complicates reproducible measurements, strongly contrasts to the advances made in the last decades on the fabrication of controllable and robust plasmonic substrates with high enhancement factors. Here, we propose an alternative approach to intracellular SERS by developing planar, nanostructured microcoins which enable to use top-down fabricated substrates for intracellular applications. We develop a nanosphere-lithography based technique for the fabrication of gold-nanotriangle and -nanodome structured silicon-nitride micron-sized chips (micro-coins). These microcoins can be lifted off form their substrate and are subsequently incorporated into fibroblast- and HeLa cells. In a model experiment, the SERS sensors are used for the label-free detection of extraneous molecules in live cells. The use of reproducible SERS substrates for intracellular sensing is expected to be an important step towards reproducible and quantitative live-cell, label-free measurements.
\end{abstract}

Keywords - Surface-enhanced Raman spectroscopy; Plasmonics, Label-free sensing

\section{INTRODUCTION}

In the last decade, a variety nanophotonic technologies has been developed for the detection biological molecules. Successful approaches include the use of refractive index sensing for a label-free peptide detection on an integrated silicon photonic platform[1], on-chip spectroscopy for the detection of spectral fingerprints through Raman spectroscopy[2], or the fabrication of reproducible plasmonic substrates for surface-enhanced Raman spectroscopy[3]. Very recently, the use of nanophotonics has taken a next step by exploring the world of intracellular sensing. Gomez-Martinez et al.[4] demonstrated that 'colloidal' micron-sized nanophotonic chips can be incorporated in living cells and used as in-vitro pressure sensors, while Shambat et al.[5] developed a nanobeam photonic crystal that can be inserted in single cells, with the goal of using these for the intracellular detection of specific protein bindings to the nanobeam cavity. Here, we develop a new type of micron-sized nanophotonic chip ('microcoin') for intracellular sensing. The chip contains a top- down fabricated gold nanopattern, which provides a controllable and reproducible localized surface plasmon resonance for surface-enhanced Raman spectroscopy (SERS). We use a nanosphere-lithography based fabrication scheme[6] for the cost efficient fabrication of these microcoins. They contrast strongly to colloidal gold nanoparticles[7], which are typically used for intracellular SERS because the latter suffer from spatiotemporal dynamics in the cell.

\section{RESULTS}

\section{A. Fabrication of nanotriangle coated microcoins}

Fig. 1 schematically shows the most important fabrication steps. First, a $200 \mathrm{~nm}$ thin film of silicon nitride $\left(\mathrm{Si}_{3} \mathrm{~N}_{4}\right)$ is deposited on a 4" silicon wafer. This thin dielectric $\mathrm{Si}_{3} \mathrm{~N}_{4}$ film acts as a carrier substrate for the later deposited gold pattern; hence its main function is the mechanical stability of the microcoin. Out of this wafer, $4 \mathrm{~cm}^{2}$ samples are cleaved, which are patterned with nanotriangles through nanospherelithography. Briefly, a hexagonally close-packed monolayer of polystyrene beads is created on top of the sample through spincoating. Afterwards, a gold layer is deposited on this closepacked layer, which serves as a template for deposition. After lifting off the beads, a close to uniform pattern of nanotriangles remains (Fig. 1 (2)). Because we want to use this patterned surface for intracellular sensing, it is necessary to cut microcoins out of the planar chip and release them from the substrate. For this purpose, disk-like coins are defined on the nanotriangle-coated substrate though contact lithography followed by an anisotropic reactive ion etch (Fig. 1(3)). The shape and size of these microcoins is fully controllable in this step, an identical procedure would allow to fabricate, e.g., a 2 $\mu \mathrm{m} \times 1 \mu \mathrm{m}$ rectangle. Finally, a potassium hydroxide $(\mathrm{KOH})$ etch is used to underetch the silicon substrate up to the point the chips detach from the substrate (Fig. 1(4)). To avoid the readsorption of the microcoins on the silicon substrate, the etchant with sample was centrifuged during etching. An ultrasonic bath further helps the detachment of the microcoins, but prolonged sonication was found to affect the adhesion of the nanotriangles to the chip. Afterwards the $\mathrm{KOH}$ etchant was washed out with water or ethanol for storage in at least four additional centrifugation steps 


\section{B. Optimization of the fabrication process}

While the overall process is similar to what is typically used for nanosphere-lithography fabricated substrates, the patterning and release of the microcoins induces a number additional constraints. First, a very good adhesion of the gold layer to the underlying $\mathrm{Si}_{3} \mathrm{~N}_{4}$ film is required, such that the gold nanotriangles stay adherent to the microcoin even after the last wet etching step. Here, the choice of a proper material for the adhesion layer, the shape of the adhesion layer and the $\mathrm{KOH}$ etching time are crucial parameters. The typically used sub-5 nm layer of titanium does not survive the potassium hydroxide etching[8], resulting in the detachment of the gold nanotriangles. We found nickel and chromium adhesion layers to provide a reasonably good alternative, however the hundred nanometer sized nanotriangles may still detach after longer potassium hydroxide etching (Fig. 2(b)). For this reason, the duration of the $\mathrm{KOH}$ etch should be as short as possible. This was achieved by first partially under etching the $\mathrm{Si}_{3} \mathrm{~N}_{4}$ disks with a reactive ion etch which is isotropic for silicon, using a $\mathrm{SF}_{6} / \mathrm{O}_{2}$ plasma (Fig. 1(3)). This way, a pillar of approximately 1-2 $\mu \mathrm{m}$ diameter remains under the $6 \mu \mathrm{m}$ diameter disk, which can then be removed in a $30 \mathrm{~m} \% \mathrm{KOH}$ etch of approximately
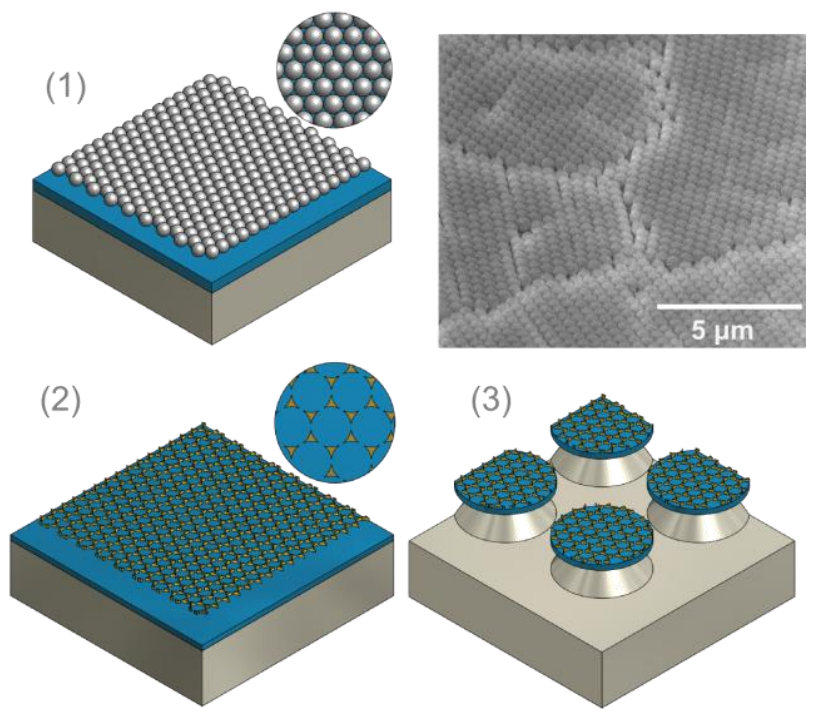

(4)
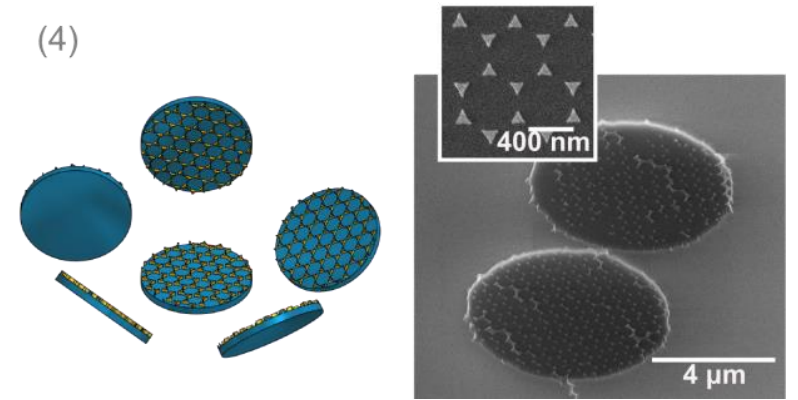

Fig. 1 - Fabrication of nanotriangle coated dielectric microdisks through nanosphere lithography for the fabrication of a nanotraingle pattern (12 ), contact lithography for the definition of indiviual chips (3) and potassium hydroxide wet etching for release from underlying substrate (4). one minute at $70{ }^{\circ} \mathrm{C}$. Finally, the adhesion of the gold nanotriangles was further improved by using a uniform adhesion layer instead of a layer which only covers the nanotriangle. This is achieved by coating the blank $\mathrm{Si} / \mathrm{Si}_{3} \mathrm{~N}_{4}$ chip with a sub-5 nm of nickel prior to the deposition of the polystyrene monolayer and the evaporation of the gold.

Apart from a good adhesion, the variability in between different nanotriangle-coated microcoins is further determined by the uniformity of the polystyrene monolayer (Fig. 1 (1)). Unavoidably, certain regions on the chip will contain double or multilayers, while other areas do not have a close-packed configuration of the beads. Furthermore, there will be line and point defects in the HCP structure. A number of different parameters are crucial in the spin coating process in order to obtain the largest possible fraction of surface area covered with a HCP-packed monolayer[9]. We achieved over $80 \%$ of HCP monolayer covered surface area when using a $5 \mathrm{w} / \mathrm{v} \%$ colloidal solution of monodisperse $448 \mathrm{~nm}( \pm 3 \%)$ polystyrene beads dissolved in a 1/2 Triton-X:Methanol (1:400) / solution. This solution of polystyrene beads was then spincoated on an oxygen plasma treated $\mathrm{Si}_{3} \mathrm{~N}_{4}$ surface at approximately 1400 rpm for 50 seconds with an acceleration of $650 \mathrm{rpm} / \mathrm{s}$. A careful optimization of spin speed, acceleration, surface treatment, surfactant and solvent ratio as well as the humidity and temperature of the environment may improve this number to over $90 \%$ of the area covered with HCP monolayers.
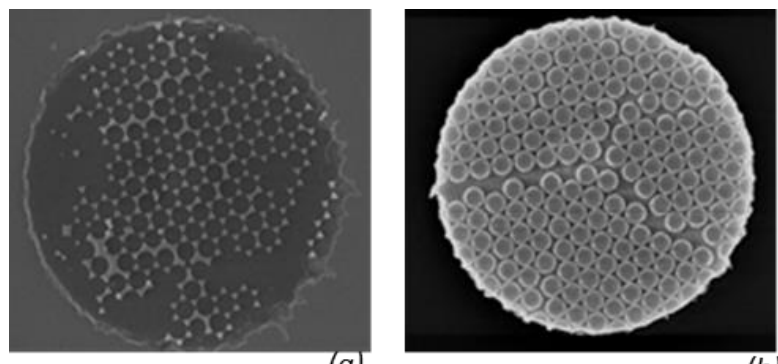

(a)

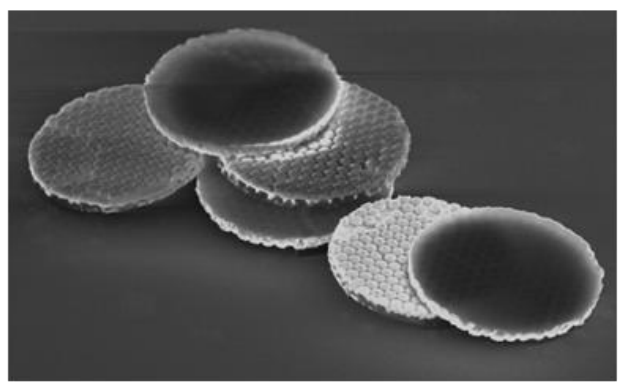

(c)

Fig. 2. SEM views of different nanostructured microcoins. (a) Nanotriangle coated chip. At several positions, the nanotriangles were not sufficiently adherent to the SiN carrier. (b) Nanodome coated chip, were the uniform gold layer suffers less from adhesion problems. (c) Side view of nanodome coated coins. (the diameter of one coin is $6 \mu \mathrm{m}$ ). 


\section{Nanodome coated microcoins.}

Starting from the monolayer of polystyrene beads, there are different possibilities for the fabrication of a nanostructured substrate for SERS. An interesting alternative to the gold nanotriangles are the so-called 'gold-film-over-nanoparticle (AuFON)' substrates[10], where a thicker gold film is directly deposited on the polymer particles without lifting off the polystyrene beads afterwards. These AuFON substrates offer an increased enhancement as compared to the nanotriangles due to a coupled localized surface plasmon resonance in the gap between the polystyrene beads and a more robust surface due to the thicker, uninterrupted gold layer, while still sharing the benefits of a nanosphere-lithography approach with respect to cost and ease of fabrication[11][12]. However, the presence of the monolayer of polystyrene beads is neither compatible with the further fabrication process, nor with the use of the microcoins for intracellular applications. Therefore, we developed an adapted fabrication scheme where the topology of the polystyrene monolayer is transferred to the underlying $\mathrm{Si}_{3} \mathrm{~N}_{4}$ substrate through reactive ion etching. This results in a nanodome patterned substrate upon which the gold film is deposited. Fig. 2 shows a SEM image of such a gold-nanodome structured microcoin. More details on the fabrication and characterization of these chips will be presented in a later publication[13].

\section{Intracellular uptake of the microcoins and intracellular SERS}

The microcoins are added to a culture of Normal Human Dermal Fibroblast cells (NHDF) in a ratio of approximately 2 particles/cells, after which they are spontaneously engulfed by the cells. After 24 hours of incubation, a large fraction $(<40 \%)$ of the coins is engulfed by the cell. The intracellular localization of the microcoins was confirmed by confocal microscopy, where the cell membrane is labelled by a green fluorescent lectin (WGA), and the microcoins are fluorescently labelled with TRITC through a silane-based linker layer (APTES) (Fig. 4).

\section{E. Characterization of the substrates.}

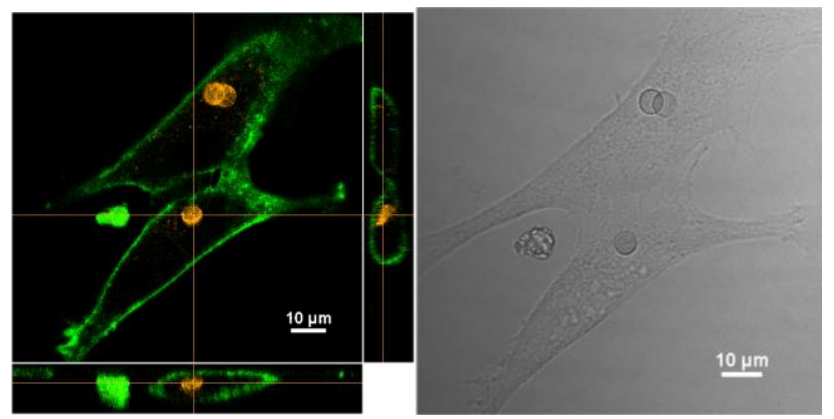

Fig. 4. Confocal microscopy cross section (left) and transmission image (rigth) showing fluorescently labelled Si3N4 microdisks in a fibroblast cell.

Fig. 3 shows a proof-of-concept experiment where the nanotriangle coated chip is used for the intracellular label-free SERS detection of rhodamine $6 \mathrm{G}$ (R6G). A $5 \mu \mathrm{M}$ solution of
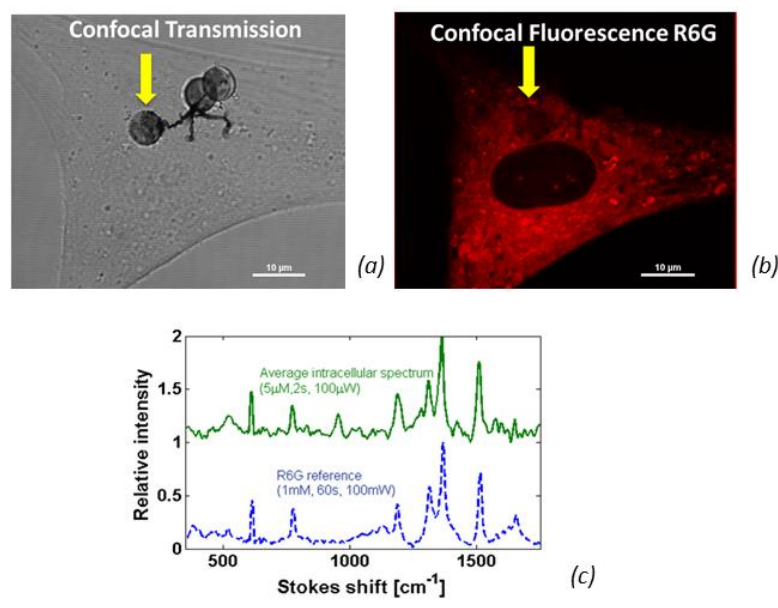

(c)

Fig. 3. Label-free SERS detection of foreign molecules, here demonstrated by a detection of rhodamine-6G. (a-b) Confocal microscopy images showing the intracellular localizatino of both the microcoin and the rhodamine, (c) SERS signal were acquired on top of the chip highlighted by the yellow arrow (green solid curve), compared to the reference spectrum of R6G (blue dashed curve)

R6G was added to the cell culture medium, after which the SERS spectrum of this molecule was detected on top of a microcoin inside the cell. The presence of R6G in the cell was further confirmed by confocal fluorescence microscopy.

\section{DISCUSSION AND CONCLUSION}

We demonstrated the fabrication of gold nanostructured microcoins, patterned through nanosphere lithography. Two different kinds of chips were presented, gold nanotriangle- and nanodome coated structures. The nanotriangle microcoins are transparent, such that they can be used for upright and inverted SERS, and offer easily accessible hotspots. The nanodomes provide a stronger enhancement and a more robust surface. The choice of a particular gold nanostructure can be application dependent, and the large variation in existing SERS substrates can provide inspiration for the future fabrication of other nanostructures on the microcoins. The possibility of using these microcoins for intracellular measurements is expected to enable more quantitative SERS experiments in the future.

\section{ACKNOWLEDGMENT}

P. C. Wuytens thanks Liesbet Van Landschoot for her help with the Scanning Electron Microscope and Steven Verstuyft for his help with cleanroom processing.

\section{REFERENCES}

K. De Vos, I. Bartolozzi, E. Schacht, P. Bienstman, and R. Baets, "Silicon-on-Insulator microring resonator for sensitive and labelfree biosensing.," Opt. Express, vol. 15, no. 12, pp. 7610-7615, 2007.

A. Dhakal, A. Z. Subramanian, P. C. Wuytens, F. Peyskens, N. Le Thomas, and R. Baets, "Evanescent excitation and collection of spontaneous Raman spectra using silicon nitride nanophotonic waveguides," Opt. Lett., vol. 39, no. 13, pp. 4025-4028, 2014. 
[3] J. J. Giner-Casares and L. M. Liz-Marzán, "Plasmonic nanoparticles in 2D for biological applications: Toward active multipurpose platforms," Nano Today, vol. 9, no. 3, pp. 365-377, 2014.

[4] R. Gómez-Martínez, A. M. Hernández-Pinto, M. Duch, P. Vázquez, K. Zinoviev, E. J. de la Rosa, J. Esteve, T. Suárez, and J. a. Plaza, "Silicon chips detect intracellular pressure changes in living cells," Nat. Nanotechnol., vol. 8, pp. 517-521, Jun. 2013.

[5] G. Shambat, S.-R. Kothapalli, J. Provine, T. Sarmiento, J. Harris, S. S. Gambhir, and J. Vučković, "Single-Cell Photonic Nanocavity Probes.," Nano Lett., vol. 13, no. 11, pp. 4999-5005, Feb. 2013.

[6] J. C. Hulteen and R. P. Van Duyne, "Nanosphere lithography: A materials general fabrication process for periodic particle array surfaces," J. Vac. Sci. Technol. A Vacuum, Surfaces, Film., vol. 13, no. 3, pp. 1553-8, May 1995.

[7] J. Kneipp, H. Kneipp, B. Wittig, and K. Kneipp, "Novel optical nanosensors for probing and imaging live cells," Nanomedicine, vol. 6, no. 2. Elsevier Inc., pp. 214-226, 2010.

[8] K. R. Williams, K. Gupta, and M. Wasilik, "Etch rates for micromachining processing - Part II," J. Microelectromechanical Syst., vol. 12, no. 6, pp. 761-778, 2003.
[9]

P. Colson, R. Cloots, and C. Henrist, "Experimental design applied to spin coating of 2D colloidal crystal masks: a relevant method?," Langmuir, vol. 27, no. 21, pp. 12800-6, Nov. 2011.

[10] D. a Stuart, C. R. Yonzon, X. Zhang, O. Lyandres, N. C. Shah, M R. Glucksberg, J. T. Walsh, and R. P. Van Duyne, "Glucose sensing using near-infrared surface-enhanced Raman spectroscopy: gold surfaces, 10-day stability, and improved accuracy.," Anal. Chem., vol. 77 , no. 13 , pp. 4013-9, Jul. 2005.

[11] P. L. Stiles, J. a Dieringer, N. C. Shah, and R. P. Van Duyne, "Surface-enhanced Raman spectroscopy.," Annu. Rev. Anal. Chem., vol. 1, pp. 601-26, Jan. 2008.

[12] C. Farcau and S. Astilean, "Mapping the SERS Efficiency and HotSpots Localization on Gold Film over Nanospheres Substrates," $J$. Phys. Chem. C, vol. 114, no. 27, pp. 11717-22, Jul. 2010.

[13] P. C. Wuytens, A. Z. Subramanian, W. H. De Vos, A. G. Skirtach and R. Baets, "Gold-nanodome patterned microchips as reproducible sensors for intracellular surface-enhanced Raman spectroscopy," Unpublished. 